\title{
DEFINITIONS AND ATTRIBUTES OF SMART HOME APPLIANCES
}

\author{
Kim, Sojung (1); Baek, Joon Sang (2) \\ 1: UNIST; 2: Yonsei University
}

\begin{abstract}
The status of Smart Home Appliances (SHAs) in the smart home industry has been raised as major components of a smart home. To design a good SHA, diverse stakeholders in the smart home ecosystem need to have a common understanding of smart homes and SHAs. However, the studies on what they are, especially those reflecting practitioners' point of view, have been scarce.

In this research, we conducted systematic literature reviews and practitioner interviews using the concept mapping method to identify the attributes of SHAs depending both on academia and industry. In addition, we suggest a framework of the smart home ecosystem which describes how those attributes are revealed in the relationships among SHAs, users, and external stakeholders.

Since all of the interviewees are Korean SHA development professionals, the findings from this research could have been limited to the current status of the Korean SHA industry. Nonetheless, we expect that this research would contribute to building a consensus on what smart homes and SHAs are and provide the designers and developers of SHAs with systematic perspectives on smart homes.
\end{abstract}

Keywords: Smart home, Smart home appliance (SHA), Business models and considerations, User centred design, Platform strategies

\section{Contact:}

Kim, Sojung

UNIST

Creative Design Engineering

Korea, Republic of (South Korea)

sojung@unist.ac.kr

Cite this article: Kim, S., Baek, J.S. (2019) 'Definitions and Attributes of Smart Home Appliances', in Proceedings of the 22nd International Conference on Engineering Design (ICED19), Delft, The Netherlands, 5-8 August 2019. DOI: $10.1017 /$ dsi.2019.213 


\section{INTRODUCTION}

The diffusion of smart innovation has stimulated the evolution of home appliances toward smart home appliances (SHAs). Every year, global trade shows for home appliances and consumer electronics reveal the tough competition among the electronics manufacturing companies. Especially for developed countries, the "smartization" of home appliances is a promising strategy to differentiate their products (CECED 2015, Uehara 2015). Aldrich (2003, p.28) argued that consumer electronics manufacturers became the main players in the smart-home market. Traditionally, electrical equipment suppliers that provide switches, sockets, distribution boards, etc., have dominated the smart-home market. Nonetheless, the high initial investment required for smart-home construction was regarded as one of the obstacles to the popularization of the smart home. Compared to building or remodelling houses, replacing old home appliances with SHAs is a more affordable way to implement a smart home. In this context, to develop worth buying SHAs is an important mission for spreading smart homes.

In order to develop a good SHA, there needs to be a common understanding of what a "good" SHA is. The common definition is also essential to build a design theory, establish a quality model, assess product quality, and develop mutual understanding (Gutiérrez et al., 2013). Since the smart home system involves diverse stakeholders that have different perspectives (Gann et al., 1999), it is important to build a common understanding on the nature of SHAs. However, compared to the activeness of SHA industry, the research on the nature of SHAs has been scarce. To set the direction of SHA development, a deeper understanding of its identity, capabilities, and potential roles should be preceded. Accordingly, this research aims at suggesting a framework that can help better understanding of a smart home ecosystem and the characteristics of SHAs. First of all, we investigated on smart homes and SHAs through literature reviews. To complement the technology-oriented precedent studies and to reflect the latest and empirical perspective to our framework, we conducted interviews and concept mapping with the SHA development practitioners based on the outcomes from the literature reviews.

\section{LITERATURE REVIEWS}

\subsection{SHAs in a smart home}

Mühlhäuser (2008) highlighted the importance of investigating the notion of a smart environment because smart products should be defined in the context of their environment. The relationship between SHAs and the smart home in the previous literature appeals to the necessity of considering the role of SHAs in the smart home. Since the concept of the smart home was introduced into the public in 1990s, many researchers have attempted to define smart homes (Table 1). According to these definitions, an SHA is a home-appliance product that can better fulfil users' needs by providing optimized services through improved product-to-product and product-to-user interactions. In a smart home connected to the world outside, SHAs also serve as a mediator between users and external service providers. Therefore, SHAs provide value to users and stakeholders outside home through its functions which involve the interactions between users, other SHAs, and the stakeholders (Figure 1).

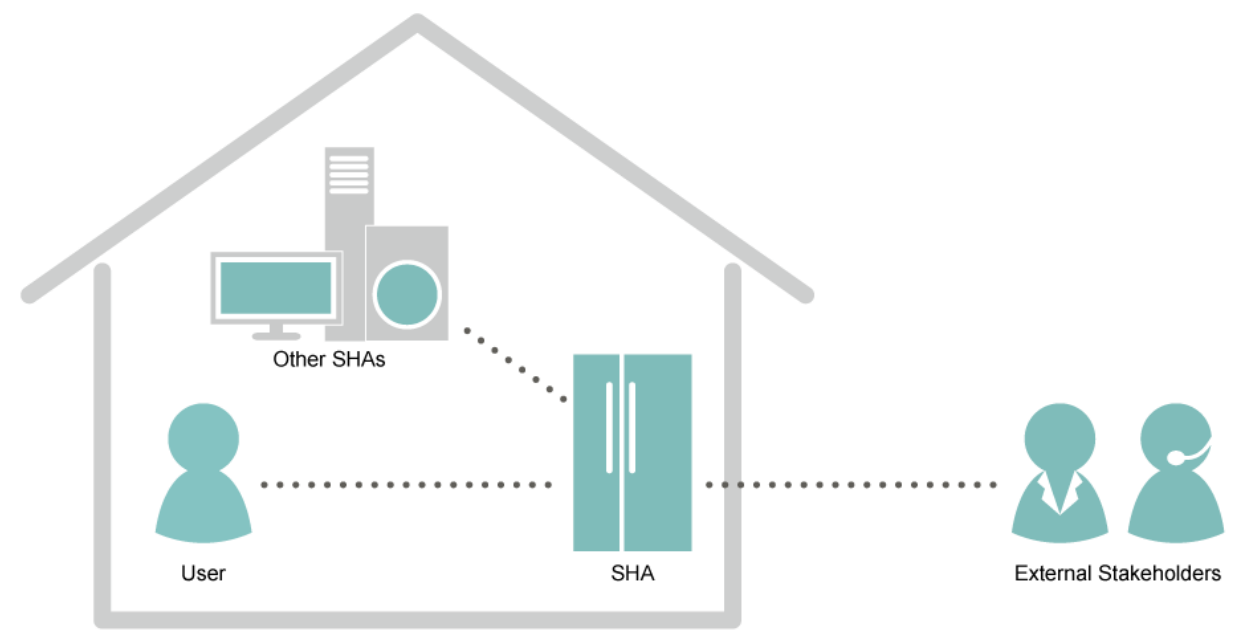

Figure 1. Relational diagram of a smart home 


\section{Definitions of smart homes}

Homes in which information and communication technologies (ICTs) have been installed to help control a variety of functions and to provide communication with the outside world.

A residence equipped with computing and information technology that anticipates and responds to the needs of the occupants, working to promote their comfort, convenience, security, and entertainment through the management of technology within the home and connections to the world beyond.

A dwelling incorporating a communications network that connects the key electrical appliances and services, and allows them to be remotely controlled, monitored, or accessed

Smart-home environments have evolved to the point where everyday objects and devices at home can be networked to give the inhabitants new means of controlling them. The smart home adjusts its functions to the inhabitants' needs in accordance with the information it collects from the inhabitants, the computational system, and the context.

\section{Reference source}

Gann et al.

$(1999$, p.1)

Aldrich

$(2003, p .17)$

Jiang et al.

(2004, p.659)

Koskela and

Väänänen-Vainio-

Mattila

(2004, p.234)

\subsection{Definitions and attributes of the smart home appliance}

The definitions of SHAs introduced in literature (Table 2) demonstrate the roles and characteristics of SHAs. They also describe the capabilities required to undertake these roles. The functional components for communication, data processing, and decision-making make the basic features of home appliances "smart." These capabilities brings the characteristics of SHAs such as contextawareness, connectivity, and automatic adjustment. As a result, SHAs can control their outputs based on the state of users, surroundings, and the data shared by other SHAs, thereby fulfilling users' needs with optimized services. To better understand the nature of SHAs, we conducted systematic literature reviews (Blizzard and Klotz 2012) and extracted the attributes of SHAs and smart products, which is an umbrella concept of SHAs. Searching in Web of Science, Scopus, and Google Scholar for the articles published since 2007, we obtained 309 items by the keywords "smart" and "appliance" and 138 items by the keyword "smart product(s)." Among them, 9 articles and 34articles articulated the attributes of SHAs and smart products respectively. We provide the list of articles containing the attributes of SHAs and smart products as an appendix. Through the inter-rater coding by 3 researchers, we integrated them into a set of SHA attributes (Table 3). Even though the outcome of the literature reviews allows us to know the attributes of SHAs better, it implies that the studies on SHAs and smart products are technology-oriented. It is in line with the finding from the research of Solaimani et al. (2015) that the major contributors of the smart home research are from the technology domain.

Table 2. Definitions of SHAs

\section{Definitions of SHAs}

Devices that are not ignorant concerning their environment and context

An appliance whose data are available to all concerned (all of the actors in the appliance life cycle) at all times throughout its life cycle

Roles: to deliver enhanced or "smart" services within the home

Home appliances that can provide customized content and smart-home service functions with network connectivity and various service control functions

A modernization of the electricity usage system of a home appliance so that it monitors, protects, and automatically adjusts its operations to the needs of its owner.

Key features: automatic adjustment, communication, and customized functions Smart appliances consist of a communication interface, a local processing and decision unit, and the appliance's actual function.

\begin{tabular}{|c|}
\hline $\begin{array}{l}\text { Reference source } \\
\text { Schmidt and Van } \\
\text { Laerhoven } \\
(2001, \text { p.66) }\end{array}$ \\
\hline $\begin{array}{l}\text { Kango et al. } \\
(2002, \text { pp. } 76,79)\end{array}$ \\
\hline $\begin{array}{l}\text { Yoon and Jang } \\
(2012, \text { p.145) }\end{array}$ \\
\hline $\begin{array}{l}\text { Association of } \\
\text { Home Appliance } \\
\text { Manufacturers } \\
(2009, \text { p.6) }\end{array}$ \\
\hline $\begin{array}{l}\text { Elmenreich and } \\
\text { Egarter } \\
(2012, \text { p.76) }\end{array}$ \\
\hline
\end{tabular}




\begin{tabular}{|c|c|}
\hline & Description \\
\hline Context-awareness & $\begin{array}{l}\text { perceive the situation, environment, and condition in which a device } \\
\text { orks }\end{array}$ \\
\hline $\begin{array}{l}\text { Knowledge } \\
\text { accumulation }\end{array}$ & $\begin{array}{l}\text { o accumulate the data related to environmental changes and users' life } \\
\text { atterns }\end{array}$ \\
\hline Inference & extract meaningful information from data analysis \\
\hline Proac & $\begin{array}{l}\text { To perform required actions without the intervention of users or a central } \\
\text { controller }\end{array}$ \\
\hline $\begin{array}{l}\text { Machine to machine } \\
\text { (M2M) cooperation }\end{array}$ & $\begin{array}{l}\text { To share capabilities and functions with other devices to achieve a } \\
\text { common goal }\end{array}$ \\
\hline M2M communication & $\begin{array}{l}\text { To share required information with other devices to achieve a common } \\
\text { goal }\end{array}$ \\
\hline Personalization & $\begin{array}{l}\text { To tailor the function of the device toward the users' purposes and } \\
\text { preferences }\end{array}$ \\
\hline $\begin{array}{l}\text { User- } \\
\text { intera }\end{array}$ & To communicate and interact with users in a natural and humanlike way \\
\hline Multifunctionality & To per \\
\hline Business-awareness & $\begin{array}{l}\text { To consider the business (business rules, business models, transaction } \\
\text { models) and legal constraints }\end{array}$ \\
\hline $\begin{array}{l}\text { Semantic } \\
\text { self-description }\end{array}$ & $\begin{array}{l}\text { To perceive the goals, plans, designs, and semantic dependencies of both } \\
\text { the overall system and each function, method, or module }\end{array}$ \\
\hline
\end{tabular}

\section{RESEARCH METHODS}

To reflect the current situation and perspectives of diverse stakeholders in SHA industry, we conducted the expert interviews with SHA-development practitioners. In the interviews, the conceptmapping method was adopted to help the interviewees organize and express their thoughts. Because of the advantages in visualization of cognitive structures, assistance in the organization of thoughts, and stimulation of the memory of related knowledge (Rye \& Rubba, 1998), concept maps can be used as an effective interview tool, especially when experts have difficulties in clarifying the implicit knowledge derived from their experiences (Novak \& Cañas, 2008). From this perspective, concept mapping was considered useful in leading the interviewees to recall the attributes of SHAs recognized in the course of their practice, and organize them in a systematic way.

The process of building a concept map was constructed based on a method described by Novak and Cañas (2008): 1) Define the focus question to be resolved through concept mapping, 2) Identify the key concepts relevant to the question domain, i.e., the attributes of SHAs in this research, 3) Organize these key concepts into a rank-ordered list from general to specific levels, 4) Reorganize them into a concept map consisting of key concepts and links showing the relationship between them, and 5) Revise the key concepts and the concept map iteratively until the concept map is sufficiently developed to answer the focus question.

As the key concepts, we utilized the attributes of SHAs (Table 3). The interviews began with the questions concerning practical experiences with SHA development and personal definition of SHAs. In the next session, the interviewees were given the key-concept cards of SHA attributes and asked to organize them into a concept map. They were allowed to freely add, remove, or revise the key concepts to describe the characteristics of SHAs as they understood. The key concept added by the interviewees are indicated in orange colour in the centre of Figure 2. In addition, to ensure the time efficiency of the interviews, the relationship between key concepts was verbally described.

The interviews lasted for 60 to 90 minutes and were recorded for transcription. The transcriptions were segmented, coded, and analysed through a thematic coding method (Galletta, 2013). Any patterns on the maps were sought and analysed in a qualitative fashion, and insights were drawn through an internal discussion. 


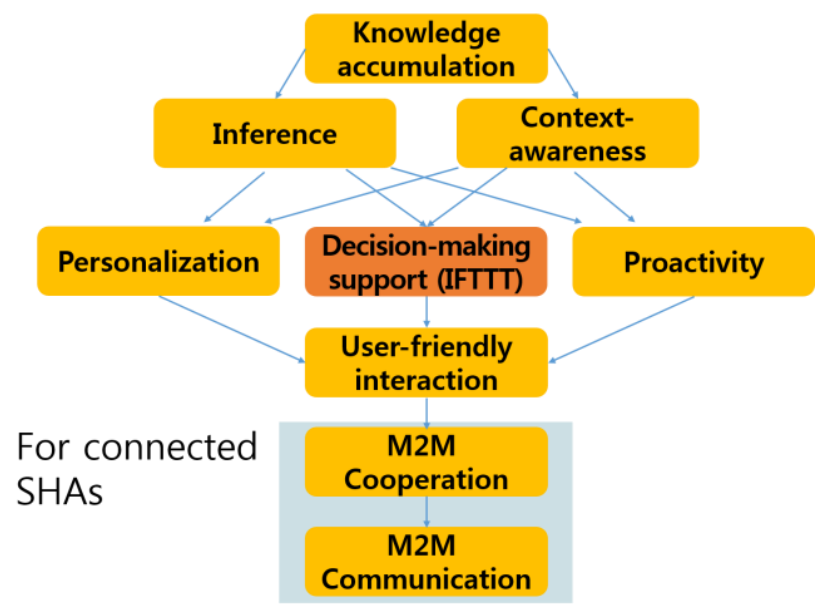

Figure 2. Example of a concept map (Digitalized)

The interviews were conducted with experts that had experience in SHA development. Seven interviewees were selected from diverse backgrounds ranging from engineering to service design to reflect broad perspectives. Their jobs range from trend research, concept generation to technology development for system implementation (Table 4). All of the interviewees were or had been SHA practitioners with the experience of developing SHAs in global companies located in South Korea and an understanding of the situation pertaining to the wider global SHA industry.

Table 4. Interviewee Information

\begin{tabular}{|l|l|l|}
\hline Affiliation and position & Role in practice & Code \\
\hline $\begin{array}{l}\text { Former) Team manager of a service design } \\
\text { agency }\end{array}$ & $\begin{array}{l}\text { User experience service design concept } \\
\text { generation for connected \& convergence } \\
\text { homes }\end{array}$ & A \\
\hline $\begin{array}{l}\text { Professor in a department of mechanical } \\
\text { design engineering/Director of a smart home } \\
\text { demonstration project }\end{array}$ & $\begin{array}{l}\text { Technology development for home } \\
\text { networks \& home solutions }\end{array}$ & B \\
\hline $\begin{array}{l}\text { Professor in charge of industry-university } \\
\text { collaboration }\end{array}$ & $\begin{array}{l}\text { Technology development for home } \\
\text { networks and energy management } \\
\text { systems }\end{array}$ & C \\
\hline $\begin{array}{l}\text { Senior designer in a home-appliance } \\
\text { company }\end{array}$ & $\begin{array}{l}\text { Trend research for drawing a smart-home } \\
\text { roadmap }\end{array}$ & D \\
\hline $\begin{array}{l}\text { Professor in a department of electrical and } \\
\text { computer engineering/Director of a ubiquitous } \\
\text { convergence research institute }\end{array}$ & $\begin{array}{l}\text { ICT development for smart home services } \\
\text { Chief researcher of a home-appliance } \\
\text { company }\end{array}$ & E \\
\hline $\begin{array}{l}\text { Director of the convergence \& standardization } \\
\text { department of the Korea Association of Smart } \\
\text { Homes }\end{array}$ & $\begin{array}{l}\text { Research and development for planning, } \\
\text { implementing, and standardizing SHAs }\end{array}$ & G \\
\hline
\end{tabular}

\section{RESULTS}

\subsection{Definition of SHAs}

Seven interviewees provided their own definitions of SHAs by identifying the value proposition, product behaviours, and technologies used to provide services (Figure 3). In terms of value propositions, the practitioners mentioned both specific offerings, such as energy management, and ultimate ones, such as convenience in life. These values should be provided naturally in the use context of home appliances (Interviewee A). Network connectivity is the primary factor to differentiate SHAs from conventional home appliances. By having a connection with other appliances, an SHA can organically cooperate with them as an agent of a converged home appliance system. Moreover, SHAs must be linked to external stakeholders if they require external resources to provide 
services. Until now, however, connectivity is seen by customers as an unnecessary add-on feature because its advantages are unclear (Interviewee F). Interfaces to communicate with users and data storage for user learning are also important (Interviewee D). As an SHA learn about its users, it adjusts its functions to its users' preferences automatically, and usability is thus improved. Since the values, product behaviours and technologies are in line with the roles, characteristics and capabilities respectively, the definitions of SHAs made by the practitioners make those from the literature reviews richer.

Smart Home Appliances perform the role in offering...

\begin{tabular}{l|}
\hline Values \\
- Energy/electricity management \\
- Information/content \\
- Better usability and performance \\
of original functions \\
- Convenience in life \\
\hline
\end{tabular}

through providing services showing..

\begin{tabular}{l} 
Product behaviours \\
- Automatically \\
- Organically \\
- By learning users' preferences \\
- In consideration of the context \\
\hline
\end{tabular}

Figure 3. Summary definition of an SHA using...

Technologies
- Network connectivity
- (to other SHAs /
external stakeholders)
- Interface
- Data storage

\subsection{Attributes of SHAs}

The interviewees articulated the attributes of SHAS which they perceived through their practices. Among a total of 11 attributes, practitioners did not provided notable opinions on knowledge accumulation, inference and personalization, but they suggested the significant changes or the issues in the other 8 attributes.

\subsubsection{Context awareness}

Contextual information is classified into two types: external information detected by external sensors or delivered by other devices, and internal information concerning the operational state of the device itself (Interviewee B). The existing definition of context awareness includes awareness of situations, the environment, and conditions. On top this, the interviewees A and B added the awareness of users: the contextual information related to personal traits of users, such as their physical and mental condition, and the interaction between users and devices, such as product usage patterns. In addition to external information concerning the environment and users, internal information should be taken into account in order to support seamless cooperation between SHAs (Interviewee B).

\subsubsection{User-friendly interaction}

Interaction is a function manifested at the touchpoints between a SHA and a user in the front stage. It is thus regarded as a factor that differentiates SHAs from conventional electronics or services and assigns novelty to them (Interviewee A). One of the objectives of user-centred interaction design is to create convenient, simple, and easy-to-use form of interactions for users (Interviewee G). To achieve this objective, interactions with SHAs need to take into account with users' mental model about physical interactions (Interviewee A). For example, consumers' intuitive understanding of the functions of play ( ) and stop ( $\mathbf{-}$ ) buttons would be applicable to the icons on touchscreens. Moreover, user experience may also change based on interaction methods. For instance, numerical data arranged in rank order would stimulate sense of competition more than those passed on simply through messages. Therefore, an appropriate interaction method should be selected while considering the objective of the interaction and the tendencies of the user (Interviewee E). To achieve humanlike and natural interaction, an in-depth study on sensitivity, i.e., the understanding of how the user accepts the interaction depending on culture, trends, and products, is necessary (Interviewee B).

\subsubsection{Proactivity}

Literature defines autonomy as the capability of SHA to function independently without external involvement. According to the practitioners, however, the current industry trend is a passive proactivity wherein the user makes decisions. One of the reasons for this tendency is resistance from 
users to the autonomous operation of these devices. Furthermore, experts predict that it would take a while before the big-data analysis technology becomes mature enough for SHAs to be sufficiently autonomous (Interviewee F). If the autonomy of the devices results in an operation that occurs in an unexpected manner or without the recognition of the user, this may cause dissatisfaction (Interviewee B). Moreover, the freedom of the user decreases as automation or personalization is enhanced. As a result, it hinders the user from identifying any problems that arise or solving these problems by themselves (Interviewee A). Therefore, practitioners believe that it is ideal for users to retain the overall ability to control the device, and apply proactivity partially to the particular services in order to provide new experiences while avoiding users' frustration.

\subsubsection{Multifunctionality}

Multifunctionality originally indicates the ability of a single product to perform multiple functions. However, the majority of the interviewees claimed that the capability of multiple SHAs to cooperate with each other and achieve additional functionalities should be emphasized. In this context, some interviewees suggested the notion of 'distributed multifunctionality': an extension of cooperation between devices and not as a single device (Interviewees B and E). For example, if a robot vacuum cleaner with human detection function cooperate with smart lightings, they can provide energy saving service by switching off the light autonomously when there is no person in the room. In short, the subject of multifunctionality is evolving from a product-level to system-level feature (Interviewee F).

\subsubsection{Semantic self-description}

To achieve smooth cooperation, SHAs need to identify its own position and abilities and as well as those of other devices (Interviewee B). In this regard, the semantic self-description, which is a basic plan for individual devices that make up the smart home, was seen as the highest-order concept and the foundation of the SHA (Interviewee A). Moreover, some interviewees regarded it as the element that differentiates home automation from the smart home. While home automation automatically operates based on a pre-set protocol, a smart home can reformulate the protocol based on the priorities of users' values. For instance, multiple control target values - such as the targets of "energy savings" and "maintaining inside temperature" - can conflict with each other. In this situation, a smart home is able to consider the priorities of the targets or the hierarchies of the SHA, flexibly reform the protocol, and make decisions. Semantic self-description is expected to form the basis for the inference to be made at system level (Interviewee D).

\subsubsection{Machine-to-machine (M2M) communication and cooperation}

Initially, M2M communication and cooperation have been introduced as separate concepts. However, interviewee $\mathrm{A}, \mathrm{E}$ and $\mathrm{F}$ suggested to combine the two concepts into one since the communication between the devices is based on intention to cooperate.

\subsubsection{Business awareness}

The interviewees tended to either exclude business awareness in concept mapping (Interviewees A and F) or place it away from the other key concepts (Interviewees B, D and E). This indicates that business awareness is too general to be seen as a unique characteristic of SHA (Interviewee A), or that it is a different-level concept compared to other attributes (Interviewee E and F). Besides, it was also claimed that business awareness encompasses too broad scope compared to other key concepts (Interviewee D). However, the interviewees recognized that business and political issues must be considered because home electronics, including SHAs, are used in close relationship with people in private places, namely, the "home." (Interviewee A). In conclusion, it is necessary to specify the concept so that it can represent an issue that occurs in the SHA industry as its specific characteristic.

\section{SMART-HOME ECOSYSTEM FRAMEWORK}

Based on the relational diagram (Figure 1) from the literature studies, interview results and concept mapping, a smart-home ecosystem involving SHA attributes was modelled (Figure 4). In this framework, M2M communication and cooperation were merged into one attribute. We also replaced multifunctionality with it because the majority of practitioners perceived it as an attribute 
collaboration between different devices rather than an attribute of a single device. Moreover, we reduced the scope of business-awareness into openness and collaboration to reflect the trend of increasing collaboration across businesses and the open platforms that support them in the SHA industry.

The attributes of smart appliances were largely divided into (a) the smartness within the appliance; (b) the ability to gather data and information from the user, other devices, and external stakeholders; (c) attributes relating to the relationship with the user; (d) those relating to the relationship between other devices and sensors; and (e) those relating to communication with other devices and sensors, and with the outside of the smart home.

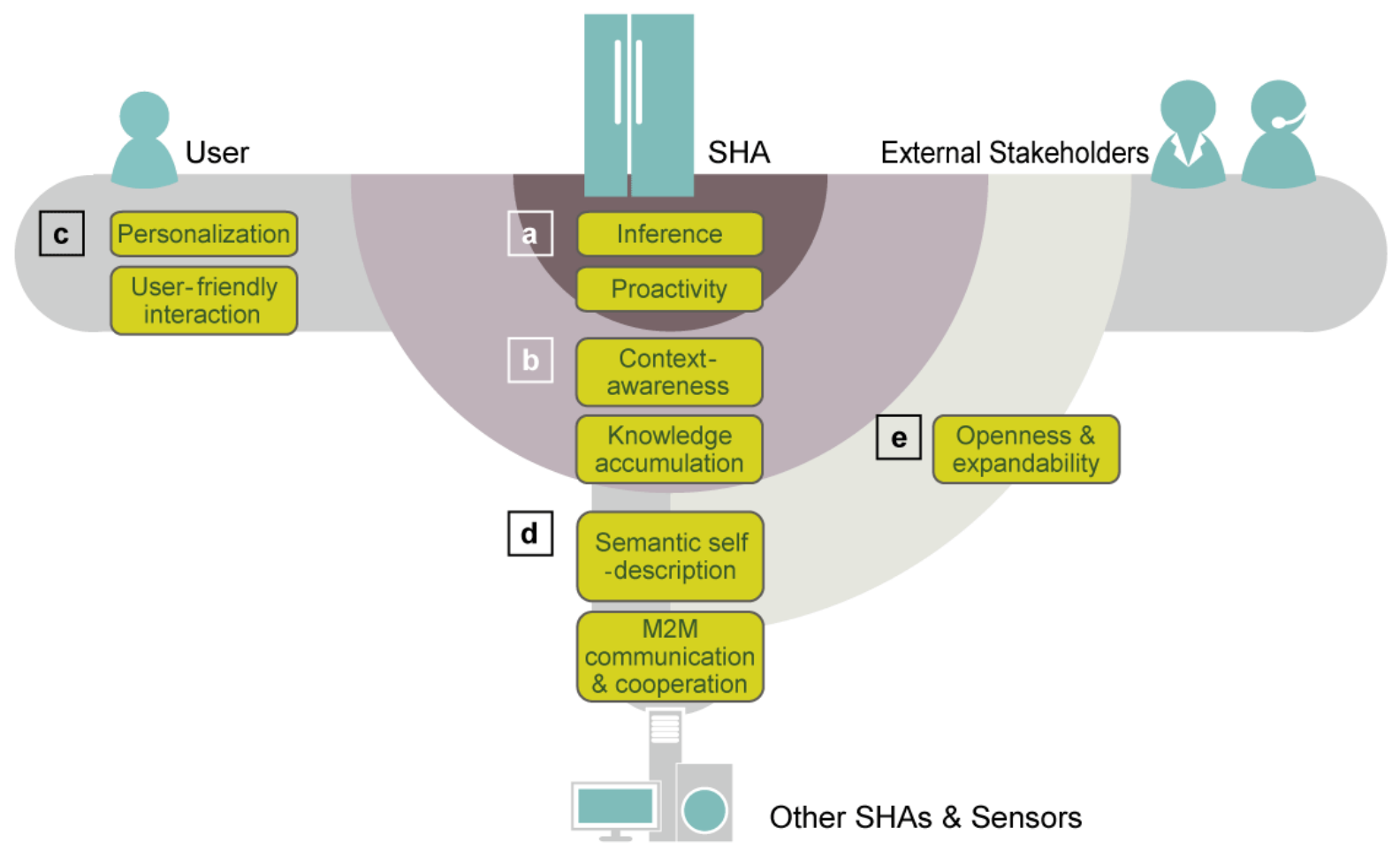

Figure 4. A framework of the smart home ecosystem

\section{1 (a) The smartness within the appliance}

The "smartness" within the SHA is an ability to process information and make decisions, and include inference and proactivity. While this will be further discussed in (b), a SHA can receive shared data or information through Internet connections from other appliances and external information sources. Additionally, it is able to gather data from the sensor on the appliance itself. These data are analysed to identify the current situation, predict future changes in situations, and autonomously search for an appropriate response.

\section{2 (b) The ability of the smart appliance to gather data and information from the user, other devices, and external stakeholders}

Considering the definitions of the data, information, and knowledge (Bellinger, Castro, \& Mills, 2004), data are symbols that represent the state of users, the environment, and devices; information is processed data that can be referred to for providing smart services; and knowledge is the patterns of user behaviour, environmental change, and device operation obtained through the application of data and information. As such, context awareness refers to the gathering of the data required and deducing contextual information, and knowledge accumulation indicates the storing of information gained from an autonomous analysis of data, or information that has been gathered externally. For example, through the power and temperature data of an oven, the contextual information of "cooking in progress" can be deduced. Then, if information on the history of oven use is gathered, a pattern for action, such as "the user typically utilizes the oven in the evenings on weekends" can be identified. Information or data on the user, the SHA itself, or other devices in the surrounding environment and 
external stakeholders can be acquired through the use of various methods. That is, a SHA can acquire data from a range of sources, from the internally included sensors in the SHA itself to the information source outside the smart home.

\section{3 (c) Attributes relating to the relationship with the user}

Interactions between the SHA and the user (e.g., user commands and information inputs to the SHA, the passing of information, or the SHA's supporting of the user's decision-making) should be designed in a user-friendly manner by considering the specific use patterns, the purpose of the interaction, and the usability. Personalization enables a SHA to control functions and provide services based on the usage purposes and user preferences. So far, it has been mainly discussed by developers in terms of content, such as "to what degree should the temperature or the moisture level be modified?" or "which content should be recommended?" However, acceptance of the proactivity of SHA, or autonomous participation by the SHA in controlling functions or providing services, varies according to the user. Specifically, extreme proactivity that does not match user intentions can decrease the level of satisfaction with SHA usage. Therefore, the application of personalization should include consideration of the level of proactivity preferred by the user.

\section{4 (d) Attributes relating to the relationship between other devices and sensors}

If two or more SHAs are connected via a network and engage in M2M communication and cooperation, they can share their sensing abilities and functions with other devices. With expanded range of sensing and influence, they are able to provide an increased level of usability and convenience. For smooth cooperation between SHAs, the goals, priority, and capabilities of entire smart homes, the individual SHAs, and its functions should be clearly defined and noted by each SHA through semantic self-description. However, it is rare to find one manufacturer that produces all SHA products, ranging from large to small appliances. Besides, the majority of homes use appliances from various manufacturers. Semantic self-description should thus be built based on agreements and cooperation among diverse manufacturers.

\section{5 (e) Attributes relating to communication with other devices and sensors and with the outside of the smart home}

Following the opinion that business awareness includes a broad range of issues, openness and expandability was suggested by the researchers as a specified concept for SHA industry. Primarily, this concept indicates "opening the smart-home platform" and "expanding the horizons of business opportunities." The success of the smart appliance business depends on the value of SHAs, i.e., the benefits attractive enough for users to pay additional costs to use SHAs. Nevertheless, there are limitations to providing value to users with previous SHA products focused on large appliances. The smart-home platform should be open and shared so that various products, including small appliances and furniture, can participate freely in M2M communication and cooperation. The industry should also be more open to external service providers and stakeholders in the production, distribution, and sales of products. By accepting a role as the medium between those actors and the user, smart appliance manufacturers, as well as external stakeholders, will be able to find new business opportunities.

\section{CONCLUSION}

The smart-home ecosystem framework supports the designers to perceive smart appliances not as a mere produce but as a component of a larger system. It also explains the relationships between appliances, users, and external stakeholders in terms of the attributes of SHAs. Considering how these relationships must be improved in the design of smart appliances would provide them a new design perspective.

To effectively research the perceptions of practitioners on the attributes of SHAs, this study conducted interviews that utilized concept mapping. The acts of defining concepts with keywords and visualizing the inter-conceptual relationships through concept mapping helped the practitioners organize their vague understanding obtained through their own work into a clear and comprehensive framework. Since this study conducted interviews with Korean SHA development professionals, the research results may reflect material that is limited to the current status of the Korean SHA industry. 
Nonetheless, home appliances are globalized products (Grinin et al., 2012) and they usually are designed through standardized approaches (Lee and Bain, 2016), so the findings from this research is valid across the nations.

\section{REFERENCES}

Aldrich, F. K. (2003), "Smart homes: past, present and future", Harper, R. (Ed.), Inside the smart home, Springer, pp. 17-39.

Association of Home Appliance Manufacturers. (2009), Smart Grid White Paper - The Home Appliance Industry's Principles \& Requirements for Achieving a Widely Accepted Samrt Grid. Retrieved from http://www.aham.org/ht/a/GetDocumentAction/i/44191

Bellinger, G., Castro, D. and Mills, A. (2004), Data, information, knowledge, and wisdom, Retrieved 25. November, 2015, from http://www.systems-thinking.org/dikw/dikw.htm

CECED. (2015), Home Appliance 2025: A Vision For The Home Appliance Industry In Europe, CECED.

Elmenreich, W. and Egarter, D. (2012), "Design guidelines for smart appliances", Presented at the WISES 2012 Proceedings, Workshop on Intelligent Solutions in Embedded Systems.

Galletta, A. (2013), Mastering the Semi-Structured Interview and Beyond: From Research Design to Analysis and Publication, NYU Press.

Gann, D., Barlow, J. and Venables, T. (1999), Digital Futures: making homes smarter, Citeseer.

Grinin, L., Ilyin, I. and Korotayev, A. (2012), Globalistics and Globalization Studies, Uchitel, Volgograd.

Gutiérrez, C., Garbajosa, J., Diaz, J. and Yagüe, A. (2013), "Providing a consensus definition for the term "Smart Product"', Presented at the 20th IEEE International Conference and Workshops on the Engineering of Computer Based Systems (ECBS).

Jiang, L., Liu, D.-Y. and Yang, B. (2004), "Smart home research", Presented at the Proceedings of the Third Conference on Machine Learning and Cybernetics, Shanghai.

Kango, R., Moore, P. and Pu, J. (2002), "Networked smart home appliances-enabling real ubiquitous culture", Presented at the 5th IEEE International Workshop on Networked Appliances, Liverpool.

Koskela, T. and Väänänen-Vainio-Mattila, K. (2004), "Evolution towards smart home environments: empirical evaluation of three user interfaces", Personal and Ubiquitous Computing, Vol. 8 No. 3-4, pp. 234-240.

Lee, D. Y. and Bain, J. (2016), "Developing a Cross-Cultural Design Methodology: A study of UK-South Korean Collaboration in Higher-Education Design", The Global e-Learning Journal, Vol. 5 No. 2.

Mühlhäuser, M. (2007), "Smart products: An introduction", Presented at the European Conference on Ambient Intelligence.

Novak, J. D. and Cañas, A. J. (2008), The theory underlying concept maps and how to construct and use them.

Rye, J. A. and Rubba, P. A. (1998), "An exploration of the concept map as an interview tool to facilitate the externalization of students' understandings about global atmospheric change", Journal of Research in Science Teaching, Vol. 35 No. 5, pp. 521-546.

Schmidt, A. and Van Laerhoven, K. (2001), "How to build smart appliances?", Personal Communications, IEEE, Vol. 8 No. 4, pp. 66-71.

Solaimani, S., Keijzer-Broers, W. and Bouwman, H. (2015), "What we do - And don't - Know about the Smart Home: An analysis of the Smart Home literature", Indoor and Built Environment, Vol. 24 No. 3, pp. 370-383. https://doi.org/10.1177/1420326X13516350

Uehara, M. (2015), "The Design of a Framework for Smart Appliances", Presented at the 29th IEEE International Conference on Advanced Information Networking and Applications Workshops (WAINA).

Yoon, M. H. and Jang, D.-H. (2012), "Current State and Development Direction of Smart Home Appliances", KEIT PD Issue Report, Vol. 12.

\section{APPENDIX}

The list of 43 analysed papers for systematic literature review is available by following the link below. https://www.slideshare.net/secret/AxwiJqcwjiTRbY

\section{ACKNOWLEDGMENTS}

This work was supported by the 'Promotion of Special Design-Technology Convergence Graduate School' of the Korea Institute of Design Promotion (N0001436), and Korea Evaluation Institute of Industrial Technology (10069188) with a grant from the Ministry of Trade, Industry and Energy, Republic of Korea 